\title{
ON A DIRECT METHOD FOR THE DETERMINATION OF AN EXACT INVARIANT FOR THE TIME-DEPENDENT HARMONIC OSCILLATOR
}

\author{
P. G. L. LEACH \\ (Received 20 July 1976) \\ (Revised 10 February 1977)
}

\begin{abstract}
An exact invariant is found for the one-dimensional oscillator with equation of motion $\ddot{q}+f(t) \dot{q}+\omega^{2}(t) q=g(t)$. The method used is that of linear canonical transformations with time-dependent coefficients. This is a new approach to the problem and has the advantage of simplicity. When $f(t)$ and $g(t)$ are zero, the invariant is related to the well-known Lewis invariant. The significance of extension to higher dimensions of these results is indicated, in particular for the existence of non-invariance dynamical symmetry groups.
\end{abstract}

\section{Introduction}

The study of the time-dependent harmonic oscillator has attracted considerable attention over the years because of interest in motion through a time-dependent electromagnetic field and the evolution of coherent states in lasers. Considerable contributions to this study have been made by Seymour [19], with others [20], Lewis $[10,11,12]$, with Riesenfeld [13], Symon [22] and Sarlet [17, 18]. Lewis used the method developed by Kruskal [6] to show that the motion described by the Hamiltonian

$$
H=\frac{1}{2} p^{2}+\frac{1}{2} \omega^{2}(t) q^{2},
$$

in which $p$ and $q$ are canonically conjugate coordinates, had as an exact invariant

$$
I=\frac{1}{2}\left[\rho^{-2} q^{2}+(\rho p-\dot{\rho} q)^{2}\right]
$$

where $\rho(t)$ was any solution of

$$
\ddot{\rho}+\omega^{2}(t) \rho=\rho^{-3} .
$$

The physical interpretation of the invariant has received attention from Eliezer and Gray [3], Günther and Leach [4] and Sarlet. Eliezer and Gray gave an angular momentum interpretation by viewing (3) as the radial component of a twodimensional oscillator motion. Sarlet followed a similar, but not so precise, line. In their extension of the discussion to the three-dimensional problem, Günther and Leach showed that the angular momentum interpretation was somewhat artificial 
and suggested that the invariant (2) was related to a time-independent Hamiltonian via a time-dependent linear canonical transformation.

It is the aim of this paper to show that the invariant is in fact a time-independent Hamiltonian in a different coordinate system. The coordinates are related via a time-dependent linear transformation. For the sake of completeness, the system considered has the Newtonian equation of motion

$$
\ddot{q}+f(t) \dot{q}+\omega^{2}(t) g=g(t)
$$

although the extension from

$$
\ddot{q}+\omega^{2}(t) q=0
$$

is essentially trivial in classical mechanics.

The use of time-independent transformations is well established. Usually they are non-linear. This causes considerable difficulty when the corresponding quantum mechanical problem is considered. Linear canonical transformations present no difficulty in quantum mechanics.

There is an extensive literature on the use of time-independent linear transformations in quantum mechanics (cf. [1,2,23]). However, time-dependent linear transformations have attracted very little attention. Pars [15] noted, in a classical context, that such may be canonical, but did not elaborate. They do not appear to have been used in quantum mechanics. In this paper, it will be seen that timedependent linear transformations are of some use. Subsequent work $[7,8,9]$ will show that they have considerable relevance to problems with time-dependent quadratic Hamiltonians.

\section{Hamiltonian formulation}

A particle of unit mass moves in one-dimensional space under the influence of the force field due to a fixed time-dependent oscillator source and is subject to a damping force linear in the velocity and a forcing term. The equation of motion is

$$
\ddot{q}+f(t) \dot{q}+\omega^{2}(t) q=g(t),
$$

where $q$ is the displacement from the source. Multiplying (6) by $\exp \{F\}$, where

$$
F=F(t)=\int_{t_{0}}^{t} f\left(t^{\prime}\right) d t^{\prime}
$$

(6) may be written as

$$
\frac{d}{d t}\left[\dot{q} e^{F}\right]-\frac{\partial}{\partial q}\left[-\frac{1}{2} \omega^{2}(t) e^{F} q^{2}\right]=g(t) e^{F}
$$

A suitable Lagrangian is

$$
L=\frac{1}{2} \dot{q}^{2} e^{F}-\frac{1}{2} \omega^{2}(t) e^{F} q^{2}+q g(t) e^{F} .
$$


That this is not unique is obvious as the ultimate term could be replaced by $-\dot{q} \int_{t_{0}}^{t} g\left(t^{\prime}\right) e^{F} d t^{\prime}$. However, (9) is sufficient for the present purpose. The momentum conjugate to $q$ is

$$
p=\dot{q} e^{F} .
$$

The Hamiltonian of the motion is therefore

$$
H=\frac{1}{2} p^{2} e^{-F}+\frac{1}{2} \omega^{2}(t) e^{F} q^{2}-q g(t) e^{F} .
$$

\section{Transformation theory}

To analyze the formalism of a linear canonical transformation between the canonical pairs $(q, p)$ and $(Q, P)$ it is convenient to write

$$
\boldsymbol{\omega}=\left(\begin{array}{l}
q \\
p
\end{array}\right), \quad \overline{\boldsymbol{\omega}}=\left(\begin{array}{l}
Q \\
P
\end{array}\right) .
$$

Hamilton's equations of motions are then

$$
\dot{\omega}=\epsilon \frac{\partial H}{\partial \omega},
$$

where $\epsilon$ is the $2 \times 2$ symplectic matrix

$$
\left|\begin{array}{cc}
0 & 1 \\
-1 & 0
\end{array}\right| \text {. }
$$

A linear transformation between $\omega$ and $\bar{\omega}$ is given by

$$
\bar{\omega}=S \omega+r \Leftrightarrow \omega=\bar{S} \bar{\omega}+\bar{r}
$$

in which $S(\bar{S})$ is a real $2 \times 2$ matrix and $\mathbf{r}(\overline{\mathbf{r}})$ a real $2 \times 1$ column matrix. The elements of both are coordinate independent, but may be time dependent. The transformation is canonical provided

$$
S \in S^{\mathbf{T}}=\epsilon .
$$

In the new coordinate system,

$$
\dot{\bar{\omega}}=\epsilon \frac{\partial \bar{H}}{\partial \bar{\omega}} .
$$

The time evolution of $\bar{\omega}$ may be studied either through (16) or through (13) and (14). As both are required to give the same description,

$$
\epsilon \frac{\partial \bar{H}}{\partial \bar{\omega}}=\dot{S} \omega+S \in \frac{\partial H}{\partial \boldsymbol{\omega}}+\dot{\mathbf{r}}
$$

In the particular case where both $H$ and $\bar{H}$ are quadratic, i.e.

$$
\left.\begin{array}{l}
H=\frac{1}{2} \boldsymbol{\omega}^{\mathbf{T}} A \boldsymbol{\omega}+\mathbf{B}^{\mathbf{T}} \boldsymbol{\omega} \\
\bar{H}=\frac{1}{2} \bar{\omega}^{\mathbf{T}} A \overline{\boldsymbol{\omega}}+\overline{\mathbf{B}}^{\mathbf{T}} \overline{\boldsymbol{\omega}},
\end{array}\right\}
$$


with $A$ and $A$ being real $2 \times 2$ symmetric matrices and $\mathbf{B}$ and $\overline{\mathbf{B}}$ real $2 \times 1$ column matrices, (17) becomes

$$
\epsilon \bar{A} \overline{\boldsymbol{\omega}}+\epsilon \overline{\mathbf{B}}=\dot{S} \boldsymbol{\omega}+S \in(A \boldsymbol{\omega}+\mathbf{B})+\dot{\mathbf{r}} .
$$

Substituting for $\bar{\omega}$ and separating the coordinate dependent and independent parts, $S$ and $\mathbf{r}$ satisfy the systems of first-order linear differential equations

$$
\begin{aligned}
& \dot{S}=\in \bar{A} S-S \in A \\
& \dot{\mathbf{r}}=\in \bar{A} \mathbf{r}+\in \overline{\mathbf{B}}-S \in \mathbf{B} .
\end{aligned}
$$

The sets of equations (20) and (21) have solutions with continuous first derivatives provided $A, \bar{A}, \mathbf{B}$ and $\overline{\mathbf{B}}$ are continuous functions of time over the interval of interest [5]. $S$ depends upon the form of $\bar{H}$ desired. This is arbitrary within the constraint of being a quadratic form. For the present discussion the signature of both $\omega^{T} A \omega$ and $\bar{\omega}^{T} \bar{A} \bar{\omega}$ is taken to be the same, viz. two. (Thus the essential nature of the problem remains unchanged. The implications of the removal of this constraint are currently under investigation.)

\section{The transformation matrix}

Bearing in mind the remarks at the end of the previous section, the transformed Hamiltonian is taken as

$$
\bar{H}=\frac{1}{2} P^{2}+\frac{1}{2} Q^{2}
$$

which is the simplest quadratic Hamiltonian of signature two. The algebra required to determine the transformation matrices is simplified if the problem is broken into several steps. The linear term in (11) does not affect the form of $S$. The intermediate form

$$
H^{\prime}=\frac{1}{2} \rho^{-2}\left(p^{\prime 2}+q^{\prime 2}\right)
$$

is introduced. Substitution of (11) and (23) into (20) yields

$$
D\left|\begin{array}{c}
y_{1} \\
y_{2} \\
\hdashline y_{3} \\
y_{4}
\end{array}\right|=\left|\begin{array}{cc:cc}
0 & \omega^{2} e^{F} & \rho^{-2} & 0 \\
-e^{-F} & 0 & 0 & \rho^{-2} \\
\hdashline-\rho^{-2} & 0 & 0 & \omega^{2} e^{F} \\
0 & -\rho^{-2} & -e^{-F} & 0
\end{array}\right|\left|\begin{array}{l}
y_{1} \\
y_{2} \\
\hdashline y_{3} \\
y_{4}
\end{array}\right|,
$$

where

$$
S=\left|\begin{array}{ll}
y_{1} & y_{2} \\
y_{3} & y_{4}
\end{array}\right|
$$

and $D$ is the operator $d / d t$. A suitable solution set of (24) is found by setting $y_{2}$ at zero, solving three of the equations in (24) to give $y_{1}, y_{3}$ and $y_{4}$ in terms of $\rho$ and then 
using the remaining equation to connect $\rho$ with $\omega^{2}(t)$ and $F(t)$. This procedure gives

$$
S=\left|\begin{array}{c|c}
\rho^{-1} e^{\frac{1}{1} F} & 0 \\
\hline\left(-\dot{\rho}+\frac{1}{2} \rho f\right) e^{\frac{1}{2} F} & \rho e^{-\frac{1}{2} F}
\end{array}\right|
$$

and $\rho$ is any twice differentiable solution of

$$
\ddot{\rho}+\left(\omega^{2}-\frac{1}{2} f-\frac{1}{4} f^{2}\right) \rho=\rho^{-3} .
$$

The matrix $S$ in (26) satisfies the condition for canonicity, viz.

$$
S \in S^{\mathbf{T}}=\epsilon \text {. }
$$

The transformation of (23) to (22) is accomplished by the matrix $R$ whose elements satisfy the set of equations

$$
D\left|\begin{array}{l}
y_{1} \\
y_{2} \\
\hline y_{3} \\
y_{4}
\end{array}\right|=\left|\begin{array}{cc|cc}
0 & \rho^{-2} & 1 & 0 \\
-\rho^{-2} & 0 & 0 & 1 \\
\hdashline-1 & 0 & 0 & \rho^{-2} \\
0 & -1 & -\rho^{-2} & 0
\end{array}\right|\left|\begin{array}{l}
y_{1} \\
y_{2} \\
\hdashline y_{3} \\
y_{4}
\end{array}\right| \text {. }
$$

The solution of this set of equations is accomplished by combining the constituent equations in pairs. The general solution is

$$
R=\left|\begin{array}{c:c}
C_{1} \cos W_{1}+C_{2} \cos W_{2} & -C_{1} \sin W_{1}-C_{2} \sin W_{2} \\
\hdashline C_{1} \sin W_{1}-C_{2} \sin W_{2} & C_{1} \cos W_{1}-C_{2} \cos W_{2}
\end{array}\right|,
$$

where

$$
W_{1}=\int_{t_{0}}^{t}\left(\rho^{-2}-1\right) d t^{\prime}, \quad W_{2}=\int_{t_{0}}^{t}\left(\rho^{-2}+1\right) d t^{\prime} .
$$

The transformation is canonical provided

$$
C_{1}^{2}-C_{2}^{2}=1 \text {. }
$$

The column matrix, r, may now be found. Writing

$$
R S=U=\left|\begin{array}{ll}
U_{1} & U_{2} \\
U_{3} & U_{4}
\end{array}\right|
$$

and substituting (11), (22) and (33) in (21), $r$ satisfies

$$
\left|\begin{array}{cc}
D & -1 \\
1 & D
\end{array}\right|\left|\begin{array}{c}
r_{1} \\
r_{2}
\end{array}\right|=\left|\begin{array}{cc}
U_{2} & g e^{F} \\
U_{4} & g e^{F}
\end{array}\right|
$$


The general solution is

$$
\left.\begin{array}{l}
r_{1}=A \cos t+B \sin t+\int_{t_{0}}^{t}\left[U_{2} \cos \left(t-t^{\prime}\right)+U_{4} \sin \left(t-t^{\prime}\right)\right] g e^{F} d t^{\prime}, \\
r_{2}=-A \sin t+B \cos t+\int_{t_{0}}^{t}\left[-U_{2} \sin \left(t-t^{\prime}\right)+U_{4} \cos \left(t-t^{\prime}\right)\right] g e^{F} d t^{\prime} .
\end{array}\right\}
$$

The explicit expressions for $U_{2}$ and $U_{4}$ are

$$
\left.\begin{array}{l}
U_{2}=\left(-C_{1} \sin W_{1}-C_{2} \sin W_{2}\right) \rho e^{-t F}, \\
U_{4}=\left(C_{1} \cos W_{1}-C_{2} \cos W_{2}\right) \rho e^{-1 F} .
\end{array}\right\}
$$

It should be noted that the generating function for the transformation from $H$ (11) to $\bar{H}$ (22) will be of the form

$$
F_{1}(Q, q, t)=a(t) Q^{2}+b(t) q^{2}+c(t) Q q+d(t) Q+e(t) q+h(t)
$$

in the case of a type one transformation.

\section{The invariant}

In the coordinate system $(Q, P)$, the Hamiltonian, $\bar{H}$, is an invariant, indeed the only non-trivial time-independent quadratic invariant, for the one-dimensional oscillator. Writing $\bar{H}$ as $I$, the Hamiltonian (11) has the invariant

$$
\begin{aligned}
I & =\frac{1}{2}[Q, P]\left|\begin{array}{l}
Q \\
P
\end{array}\right| \\
& =\frac{1}{2}[q, p] S^{\mathbf{T}} R^{\mathbf{T}} R S\left|\begin{array}{l}
q \\
p
\end{array}\right|+\mathbf{r}^{\mathbf{T}} R S\left|\begin{array}{l}
q \\
p
\end{array}\right|+\frac{1}{2} \mathbf{r}^{\mathbf{T}} \mathbf{r},
\end{aligned}
$$

where $S, R$ and $\mathbf{r}$ are given by (26), (30) and (35) respectively. The explicit expression for the invariant is rather lengthy and is omitted for the general case. The invariance of $I$ may be checked directly using

$$
I=[I, H]_{P B}+\frac{\partial I}{\partial t}
$$

the definition of $H$ and the differential equations governing the time-explicit parts of $I$.

\section{Comment}

The method used to derive the invariant (38) differs from that used by earlier writers and has certain attractive features. The method is placed firmly within the context of the central principles of Hamiltonian mechanics. The time-dependent 
Hamiltonian (11) is seen to be related via linear canonical transformations to the Hamiltonian of the simplest oscillator of all,

$$
\bar{H}=\frac{1}{2}\left(P^{2}+Q^{2}\right) \text {. }
$$

Expressed in terms of the original coordinates $\bar{H}(23)$ is the invariant $I$. This confirms the physical interpretation of the invariant suggested by Günther and Leach. The effect of the transformation has been to remove the time dependence from the Hamiltonian to the frame of reference. It is the exact analogue to the use of non-inertial frames in Newtonian mechanics.

In the particular case in which there is no forcing term $(g(t)=0)$,

where

$$
\begin{aligned}
I= & \frac{1}{2}\left\{\left(\rho^{-1} e^{\frac{1}{ \pm} F} q\right)^{2}(\cosh 2 C+\sinh 2 C \cos 2 W)\right. \\
& +\left[\rho e^{-1 F} p-\left(\dot{\rho}-\frac{1}{2} \rho f\right) e^{\frac{1}{2} F} q\right]^{2}(\cosh 2 C-\sinh 2 C \cos 2 W) \\
& \left.-2\left(\rho^{-1} e^{\frac{1}{1} F} q\right)\left[\rho e^{-1 F} p-\left(\dot{\rho}-\frac{1}{2} \rho f\right) e^{\frac{1}{2} F} q\right] \sinh 2 C \sin 2 W\right\} .
\end{aligned}
$$

$$
W=\int_{t_{0}}^{l} \rho^{-2} d t^{\prime}, \quad C_{1}=\cosh C, \quad C_{2}=\sinh C .
$$

Setting $C=0,(40)$ is essentially the invariant given by Eliezer and Gray. With the same value of $C$ and $F=0,(40)$ is exactly the invariant which Lewis derived using Kruskal's method.

More generally, for undamped and unforced motion,

$$
\begin{aligned}
I= & \frac{1}{2}\left[\rho^{-2} q^{2}(\cosh 2 C+\sinh 2 C \cos 2 W)\right. \\
& +(\rho p-\dot{\rho} q)^{2}(\cosh 2 C-\sinh 2 C \cos 2 W) \\
& \left.-2 \rho^{-1} q(\rho p-\dot{\rho} q) \sinh 2 C \sin 2 W\right] .
\end{aligned}
$$

Lewis' invariant is

$$
I=\frac{1}{2}\left[\tau^{-2} q^{2}+(\tau p-\dot{\tau} q)^{2}\right],
$$

where $\tau$ has been written instead of the usual $\rho$. At first sight (42) appears to be different from (43) since it is a three-parameter homogeneous quadratic in $q$ and $p$. As Lewis noted [11], (43) is a two-parameter form since $\tau(t)$ belongs to a twoparameter family of functions (see also Pinney [16] and Eliezer and Gray). That this is not the case is easily demonstrated. The expression (43) is (42) with $C=0$. Suppose that $C$ is given some arbitrary non-zero value. Requiring the value of $I$ to remain constant implies that

$$
\left(\ddot{\rho}+\omega^{2} \rho-\rho^{-3}\right)\left\{\dot{\rho} \cosh 2 C+\sinh 2 C\left(\rho^{-1} \sin 2 W-\dot{\rho} \cos 2 W\right)\right\}=0
$$

which is satisfied identically. Thus $\tau$ and $\rho$ satisfy the same differential equation. However, for $C \neq 0, \rho \neq \tau$. The relaxation of the requirement $I(C)=I(0)$ naturally increases the number of parameters from two to three. This point was previously 
appreciated by Eliezer and Gray where they point out that the Lewis invariant is subject to a scaling constant.

\section{Applications}

The use of invariants in the solution and discussion of problems in quantum mechanics has been well established (cf. [12], [14], [21]). Lewis has shown that an invariant may be used to solve the time-dependent Schrödinger equation for the problem. The employment of time-dependent transformations to obtain the wave function directly from that of the corresponding time-independent problem is an attractive proposition. In a subsequent paper [8], the work of Wolf [23] and Boon and Seligman [1] on time-independent problems will be extended to the present class of problems.

The existence of the invariant is of direct relevance to the question of the existence of dynamical symmetry groups. Under linear canonical transformations the dynamical symmetries of $\bar{H}(22)$ are preserved in the invariant $I$ (38). As $I$ does not commute with $\bar{H}(11)$, the symmetry group of $I$ constitutes a non-invariance dynamical symmetry group [14,21]. The existence of this symmetry group is of more interest for problems of higher dimension. In a later work $[7,9]$ the existence of an invariant for any Hamiltonian of quadratic form will be further investigated. It will be shown that, for all such Hamiltonians, there are sufficient quadratic constants of the motion which commute with the invariant to form a basis for the generators of the Lie group $S U(n)$ (for $n$-dimensional motion).

\section{Acknowledgement}

The author thanks H. R. Lewis, Jr. for his interest in the present work and for useful comments regarding the contents of Section 6.

\section{REFERENCES}

[1] M. H. Boon and T. H. Seligman, "Canonical transformations applied to the free Landau electron", J. Math. Phys. 14 (1973), 1224-1227.

[2] C. P. Boyer and K. B. Wolf, "Canonical transforms. III. Configuration and phase descriptions of quantum systems possessing an $s l(2, R)$ dynamical algebra", $J$. Math. Phys. 16 (1975), 1493-1502.

G. H. Katzin and J. Levine, "Dynamical symmetries and constants of the motion for classical particle systems", J. Math. Phys. 15 (1974), 1460-1470.

R. H. Kohler, "Extended view of classical contact transformations", Foundations of Physics 6 (1976), 193-208.

J. D. Louck, M. Moshinsky and K. B. Wolf, "Canonical transformations and accidental degeneracy. I. The anisotropic oscillator", J. Math. Phys. 14 (1973), 692-695.

M. Moshinsky, "Canonical transformations and quantum mechanics", SIAM J. Appl. Math. 25 (1973), 193-212.

M. Moshinsky and J. Patera, "Canonical transformations and accidental degeneracy. IV. Problems with continuous spectra", J. Math. Phys. 16 (1975), 1866-1875.

M. Moshinsky and C. Quesne, "Linear canonical transformations and their unitary representations", J. Math. Phys. 12 (1971), 1272-1283. 
[2] K. B. Wolf, "Canonical transforms. I. Complex linear transforms”, J. Math. Phys. 15 (1974), 1295-1301.

[3] C. J. Eliezer and A. Gray, "A note on the time-dependent harmonic oscillator", SIAM J. Appl. Math. 30 (1976), 463-468.

[4] N. J. Günther and P. G. L. Leach, "Generalized invariants for the time-dependent harmonic oscillator", J. Math. Phys. 18 (1977), 572-576.

[5] E. L. Ince, Ordinary Differential Equations, pp. 71, 72, Dover, New York (1956).

[6] M. Kruskal, "Asymptotic theory of Hamiltonian and other systems with all solutions nearly periodic", J. Math. Phys. 3 (1962), 806-828.

[7] P. G. L. Leach, "On the theory of time-dependent linear canonical transformations as applied to Hamiltonians of the harmonic oscillator-type", La Trobe University Department of Mathematics preprint (to appear in J. Math. Phys.).

[8] P. G. L. Leach, "Invariants and wave-functions for some time-dependent harmonic oscillator-type Hamiltonians", La Trobe University Department of Mathematics preprint.

[9] P. G. L. Leach, "Quadratic Hamiltonians, quadratic invariants and the symmetry group $S U(n)$ ", La Trobe University Department of Mathematics preprint.

[10] H. R. Lewis, Jr., "Classical and quantum systems with time-dependent harmonic oscillator-type Hamiltonians”, Phys. Rev. Letters 18 (1967), 510-512.

[11] H. R. Lewis, Jr., "Motion of a time-dependent harmonic oscillator and of a charged particle in a class of time-dependent axially symmetric electromagnetic fields", Phys. Rev. 172 (1968), 1313-1315.

[12] H. R. Lewis, Jr., "Class of exact invariants for classical and quantum time-dependent harmonic oscillators", J. Math. Phys. 9 (1968), 1976-1986.

[13] H. R. Lewis, Jr. and W. B. Riesenfeld, "An exact quantum theory of the time-dependent harmonic oscillator and of a charged particle in a time-dependent electromagnetic field", J. Math. Phys. 10 (1969), 1458-1473.

[14] N. Mukunda, L. O'Raifeartaigh and E. C. G. Sudarshan, "Characteristic non-invariance groups of dynamical systems", Phys. Rev. Letters 15 (1965), 1041-1044.

[15] L. A. Pars, A Treatise on Analytical Dynamics, p. 500, Heinemann, London (1965).

[16] E. Pinney, "The non-linear differential equation $y^{\prime \prime}+p(x) y+C y^{-3}=0 "$, Proc. Amer. Math. Soc. 1 (1950), 681.

[17] W. Sarlet, "Class of Hamiltonians with one-degree-of-freedom allowing application of Kruskal's asymptotic theory in closed form. I", Annals of Phys. 92 (1975), 232-267.

[18] W. Sarlet, "Class of Hamiltonians with one-degree-of-freedom allowing application of Kruskal's asymptotic theory in closed form. II", Annals of Phys. 92 (1975), 248-261.

[19] P. W. Seymour, "Motions of charged particles in plasmas", Int. J. Engng. Sci. 1 (1963), 423-451.

[20] P. W. Seymour, R. B. Leipnik and A. F. Nicholson, "Charged particle motion in a timedependent axially symmetric magnetic field", Aust. J. Phys. 18 (1965), 553-565.

[21] E. C. G. Sudarshan, N. Mukunda and L. O'Raifeartaigh, "Group theory of the Kepler problem", Phys. Lett. 19 (1965), 322-325.

[22] K. R. Symon, "The adiabatic invariance of the linear or non-linear oscillator", J. Math. Phys. 11 (1970), 1320-1330.

[23] K. B. Wolf, "Canonical transforms, separation of variables and similarity solutions for a class of parabolic differential equations", J. Math. Phys. 17 (1976), 601-613.

Department of Mathematics

La Trobe University

Bandoora, Vic. 3083

Australia 